\title{
To live in genetic diversity: wild emmer in Fertile Crescent and its use for plant breeding
}

\author{
Özkan H. ${ }^{1 *}$, Mazzucotelli E. ${ }^{2}$ \\ ${ }^{1}$ University of Çukurova, Faculty of Agriculture, Department of Field Crops, Adana, Turkey \\ ${ }^{2}$ Council for Agricultural Research and Economics (CREA): Research Centre for Genomics \\ and Bioinformatics, Fiorenzuola d'Arda (PC), Italy \\ *e-mail:hozkan@cu.edu.tr
}

The transition from hunting and gathering to agriculture had revolutionary consequences for the development of human societies. Crops such as wheat, barley, lentil, pea, and chickpea played a crucial role in the establishment of complex civilizations in southwest Asia. Wild emmer wheat (Triticum dicoccoides, $2 n=28$, BBAA) was one of the first cereal to be domesticated in the Fertile Crescent between c. 12,000 and c. 10,000 years ago. The wild emmer wheat is the progenitor of the domesticated durum wheat (AABB), and fully fertile in cross with it. This step provided the key for subsequent bread wheat evolution. Therefore, it is called as "Mother of wheat". Wild emmer is found today in the western Fertile Crescent in Jordan, Syria, Israel, and the central part of southeastern Turkey. Wild emmer harbors a wide spectrum of genes and alleles lost during domestication and breeding, nevertheless, a number of genes and alleles positively contributing to biotic and abiotic stress tolerance, yield components and quality traits have been found in wild emmer and transferred to cultivated wheats by crossing experiment. Recently, a wide collection of wild emmer accessions has been established to recover useful genetic diversity for cultivated wheats. This collection is being used for genome-wide association (GWAS) for a number of traits. In this presentation, we will discuss its domestication progress and its use for a wheat breeding program. 